\title{
Order Batch Formations for Less Picker Blocking in a Narrow-Aisle Picking System
}

\author{
Soondo Hong* \\ Department of Industrial Engineering, Pusan National University, Busan, Korea
}

(Received: July 18, 2015 / Revised: September 14, 2015 / Accepted: September 14, 2015)

\begin{abstract}
This paper analyses the best batch formations for order picking throughput in narrow-aisle order picking systems. Our analytical comparison finds that a high pick density variation leads to a heavy picker blocking. Simulation experiments show that a distance-based batching algorithm reduces picker blocking by decreasing the number of aisles visited and stabilizing the variation in number of picks per aisle by packing orders tightly, and that the solution quality and mechanism for determining the batch size dictated by the sorting strategy causes varying amounts of blocking. We conclude that combining a distance-based batching method with an appropriate batch sizing strategy will reduce picker blocking and shorten travel in narrow-aisle picking systems.
\end{abstract}

Keywords: Warehousing, Order Batch Picking, Batching and Routing, Picker Blocking, Simulation Comparison

* Corresponding Author, E-mail: soondo.hong@pusan.ac.kr

\section{INTRODUCTION}

Picking orders in batches from an order picking system (OPS) is generally favored when customers' demands create a large number of small orders. When multiple pickers are needed to obtain the required throughput in the picking process, order picking performance often declines due to picker blocking. In particular, narrow-aisle picking environments, which are attractive for their storage capability, can produce significant picker blocking even though one-way traversal routing is used to mitigate congestion (Gue et al., 2006). Popular distance-based batching approaches perform efficiently when there are a small number of pickers, but become less productive due to congestion when staffing multiple pickers for a higher demand fulfilment. For example, in Ruben and Jacobs's study (1999), order picking productivity per person declines when batching. Thus, resolving congestion is critical for improving work force utilization (Tompkins et al., 2003).

This paper examines environments with multiple narrow-aisles where the order picking is managed by distance-based order batching algorithms and the choice of a sorting strategy as well as a storage policy. We expect that when a batching algorithm aggregates orders with the same number of items to reduce the expected travel distance, the work load per unit distance will fluctuate depending on the location of the items and the length of the routes. We also expect that the choice of sorting strategy will determine the batch size, whereas the choice of storage policy (slotting strategies) will reduce expected order picking travel distance.

The previous literature on picker blocking and pick density variations tends to study them separately. Ruben and Jacobs (1999), who conduct a comparative study investigating picker blocking, do not investigate the relationship between picker blocking and batching algorithms. Picker blocking literature (Gue et al., 2006; Parikh and Meller, 2009; Parikh and Meller, 2010; Skufca, 2005) is silent on the relationship between batching and both pick density and picker blocking. Therefore, they explicitly relax the uniform pick density commonly imposed in the literature. For example, in our simulation study of narrow-aisle picking (see section 5.3), we find that batches assembled by a strategy that determines batch size by number of orders encounters less picker blocking than 
those built by a strategy in which the number of items limits the batch size, even though both batches exhibit a similar pick density. To our knowledge, this observation has not been reported.

We contribute to the literature by comparing all relevant analytical models of pick density variations and picker blocking and then recommending an efficient batching strategy (i.e., batch formation) that considers picker blocking in distance-based order batching. The remainder of this paper is organized as follows. Section 2 reviews picker blocking models in the literature. We define the picker blocking issues in batch picking and the scope of this paper in Section 3. Section 4 compares the analytical models to obtain knowledge on picker blocking in a narrow-aisle. Section 5 conducts a simulation study of batch-sizing strategies, storage policies, and order picking system layouts and determines appropriate batching strategies for increased throughput by reducing picker blocking. We summarize the findings and offer suggestions for future research in Section 6 .

\section{LITERATURE SURVEY}

\subsection{Distance-Based Batching Algorithm}

Batching algorithms help to aggregate batches in large-scale order picking situations. In general, distancebased approaches with their relatively simple implementation (Ruben and Jacobs, 1999) are preferred for aggregating orders whose items are closely located within a storage space. The literature (Ruben and Jacobs, 1999; De Koster et al., 1999; Gademann and Van de Velde, 2005; Gademann et al., 2001; Hong et al., 2012) describes various algorithms that identify orders to be picked together for the purpose of reducing travel distances and number of trips.

One popular large-scale batching algorithm is the seed algorithm, which is attractive because of its simple implementation and excellent computational efficiency (De Koster et al., 1999). In particular, De Koster et al.'s algorithm (De Koster et al., 1999) improves batching quality by using the following procedure: 1) select a seed having the largest number of aisles; 2) choose the order minimizing the number of additional aisles; and 3) update the seed when adding an order to it.

De Koster et al. (1999) also suggest that the saving algorithm is preferable to the seed algorithm. The saving algorithm uses a save list to choose a better alternative between possible batches. For example, a modified Clarke and Wright method (De Koster et al., 1999; Clarke and Wright, 1964), termed CW II, obtains a pair of appropriate batch candidates from a save list and switches orders in two candidates to reduce travel. The save list is updated until there is no saving pair left.

The route-packing based order batching procedure (RBP) algorithm in Hong et al. (2012) produces a highquality solution in a short time. It uses a packing method to group orders into appropriate batches. Their work also finds that the gap error is less than $3 \%$ in a largescale problem.

\subsection{Picker Blocking in Narrow-Aisle Picking Systems}

A narrow-aisle system is typically characterized by no-passing in an aisle. The congestion created by the nopassing condition is termed picker blocking (Figure). Several researchers (Gue et al., 2006; Parikh and Meller, 2010; Skufca, 2005; Hong et al., 2013; Hong et al., 2015a) have investigated the effects of picker blocking on order picking performance. For example, Skufca (2005) presents a $k$-picker congestion model of a circular nopassing system in the case of infinite walk speed. Gue et al. (2006) address two-picker congestion models of a parallel-aisle pick area approximated by a circular nopassing system considering infinite and unit walk speeds In the unit walk speed case, the unit walk time to pass a pick face is identical to the unit pick time. Gue et al. also conduct simulation experiments to investigate picker behavior under more practical walk speed assumptions, identifying the effects of pick density on narrow-aisle order picking performance under the single-pick assumption where a picker has at most one pick at a pick face. They conclude that a batch picking strategy in narrowaisle OPSs is advantageous when the pick density is either very low or very high.

Parikh and Meller (2010) develop two-picker congestion models under extreme walk speed assumptions where repeated picks at a pick face are allowed, which they call a non-deterministic picking situation. They find that picker blocking can be significant when there is high variation in the pick density. Their observation, however, is flawed, since they compare the amount of picker blocking over pick density, which does not stand for the same work load.

Hong et al. (2015a) show that the circular-aisle congestion model is applicable for a bucket brigade order picking situation. In order picking situations, bucket brigades provide self-organizing and self-balancing characteristics. The authors prove that when the backward walk time is instantaneous and the hand-off time is zero, the congestion model of bucket brigade order picking is equivalent to the congestion model of the circularaisle abstraction. In Hong et al. (2015b), the extension for order batching confirms the analytical model's rigid-

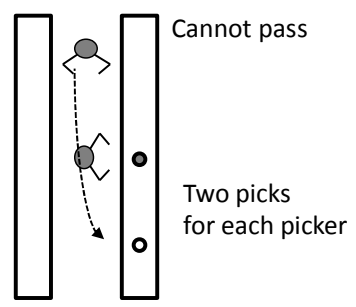

Figure 1. Picker blocking (Parikh and Meller, 2009). 
ity and the authors apply the analytical model to situations of blocking mitigation. The authors describe the development of an extreme control model in a bucket brigade order picking system.

\subsection{Picker Blocking in Distance-Based Order Batching}

Ruben and Jacobs (1999), who indicate that congestion affects the selection of batching procedures and storage policies, find that a turnover-based storage policy (popular products as measured by demand are stored in desirable locations measured by the distance from the input/output point) generates more congestion than random storage policies. In proposing a batching algorithm which minimizes travel distance, Hong et al. (2012) report a small increase in retrieval time due to picker blocking. Their resulting order-picking operations are relatively robust to picker blocking.

We note that Ruben and Jacobs (1999) do not establish a relationship between picker blocking and batching algorithms, nor do they give a clear theoretical rationale for the congestion observed. Hong et al. (2012) report limited picker blocking when their proposed batching algorithm is used to generate batches, but they do not explain why their algorithm would experience relatively little picker blocking.

\subsection{Issues}

A review of the available literature also identifies a critical issue regarding narrow-aisle batch picking: no simulation or analytical studies have fully investigated the relationship between picker blocking and the batching algorithm even though, in practice, the algorithm may have a significant effect on pick density level and variation. Parikh and Meller (2010)'s comparison of high and low pick time variations does not definitively show that a high variation over the same work load results in more picker blocking. Neither Ruben and Jacobs (1999) nor Hong et al. (2012) identify the batch formations which increase the variation of pick time.

\section{PROBLEM DEFINITION}

\subsection{Picker Blocking in Narrow-Aisle Picking Systems}

In narrow-aisle picking systems, pickers travel through one-way aisles to retrieve items from shelves and place them in a cart as schematically illustrated in Figure 2. When an aisle includes no items assigned to the picker, the aisle can be skipped to shorten the travel distance if the unidirectional characteristic of all of the aisles can still be maintained. Picker blocking occurs in an aisle when an upstream picker cannot pass a downstream picker (see Figure 1).

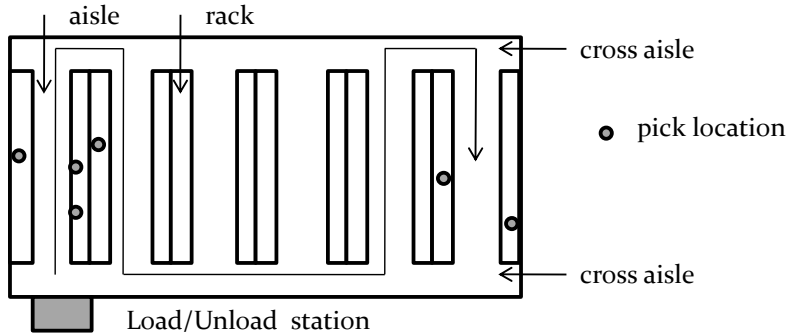

Figure 1. A Narrow-Aisle System and a Routing Example (Modified from Gademann and Van de Velde, 2005).

Order picking throughput deteriorates as much as the blocking delay. When each picker is blocked $b(k)$ fraction of the time, $0 \leq b(k) \leq 1$, the throughput is:

$$
\lambda(k)=k \cdot\left[\frac{E[p t]}{E[p t] t_{p}+t_{w}}\right](1-b(k))
$$

where $E[p t]$ represents the expected number of picks at a stop. The time to pick $\left(t_{p}\right)$ represents the average time the picker is stopped, including the time spent picking items. The time to walk $\left(t_{w}\right)$ indicates the average time to walk past a single location in the pick face.

\subsection{Batch Algorithms and Relevant Batch-Sizing and Storage Methods}

In many practical situations, order sizes are relatively small compared to cart capacity and the orders are batched to improve picking throughput. Since total retrieval time, which is dependent on the travel distance, drops when pickers collect multiple orders in the same trip, the batching algorithm has to efficiently reduce both the number of trips and the travel distance.

In addition, managers need to be able to change both the batch-sizing strategy and storage policy to maximize order picking performance. The batch-sizing strategy, which is strongly associated with sorting strategy, impacts the batching algorithm by affecting the units of measure that determine the batch size. First, the batch size is capacitated by the number of orders (i.e., ordercapacitated batch-sizing). Typically, each picker's cart carries bins or boxes that hold each order separately in a sort-while-pick operation. Thus, a batch is determined by the number of bins, i.e., the number of orders that fit on the cart. Second, each batch also can include the same number of items (i.e., item-capacitated batch-sizing). In the pick-then-sort strategy, all items are placed in a common bin and later separated into orders in a secondary sorting operation. The maximum batch size is determined by the number of items that fit on the cart.

Storage policies (i.e., slotting strategies) also affect picker blocking. Dedicated or class-based storage policies that store the more frequently requested items closer to the loading station increase pick density in these areas, 
which tends to increase picker blocking.

Finally, picker blocking $(b(k))$ is associated with the various impacts of batching algorithms, batch-sizing strategies, and storage policies upon batch formations. Yet, the batching algorithms now used only optimize the distance-related component of the objective function and the storage policy is associated with picker blocking. However, impacts by batching solution quality and batchsizing strategy are not studied well.

\subsection{Scope of Study}

First, we compare less pick and heavy pick variation cases over $E[p t]$ from models in the literature. Second, based on the knowledge gained we examine batch formations and picker blocking under different batching algorithms, batch-sizing strategies, and storage policies. We focus on large-scale batching algorithms because picker blocking is a concern in large-scale situations.

\section{ANALYTICAL COMPARISON IN A CIRCULAR-AISLE SYSTEM}

This section examines the impacts on picker blocking from the variations in the number of picks at a stop. We consider the circular-aisle abstraction and review the analytical picker blocking models. We then define an equivalent work load condition, conduct the analytical comparison results, and draw new insights.

\subsection{A Circular-Aisle Abstraction and Picker Blocking Models}

To simplify the analysis of the picker blocking phenomena, a picking system with multiple narrow aisles is often modelled as a single circular order picking aisle as shown in Figure 3. This abstraction was introduced by Skufca (2005) and applied by Gue et al. (2006), Parikh and Meller (2010), and Hong et al. (2013) for a narrowaisle system. The circular order picking aisle consists of $n$ pick faces. Pickers move only in a clockwise direction, meaning that they travel through the aisle in only one direction. Pick time is constant regardless of the pick face characteristics such as shelf height. At a pick face,

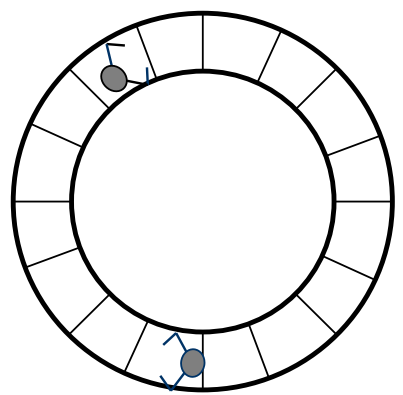

Figure 3. A circular order picking aisle (Gue et al., 2006).
Table 1. The Percentage of Time Blocked when Two Pickers Work ( $p=$ pick density, $n=$ the number of pick faces)

\begin{tabular}{ccc}
\hline & Pick: walk time & Analytical models \\
\hline Single-pick & $1: 1$ & $\frac{p(1-p)}{(n-1)(p+1)^{2}-2 p^{2}}$ \\
& $1: 0$ & $\frac{1-p}{2(1-p)+(n-1) p}$ \\
\hline Multiple-picks & $1: 1$ & $\frac{p}{2 p+n-1}$ \\
& $1: 0$ & $\frac{1}{2+(n-1) p}$ \\
\hline
\end{tabular}

pickers pick with a probability $p(q=1-p)$. The pick time, $t_{p}$, and the walk time between two pick faces, $t_{w}$, are deterministic.

There are two restrictive cases of pick: walk times: 1) walk speed is equal to unit pick time per pick face (pick: walk time $=1: 1$ ); and 2) walk speed is infinite (pick: walk time $=1: 0$ ). As a performance measure, the percentage of time blocked has been evaluated, denoted as $b_{t p: t w}^{s}(2)$ and $b_{t p: t w}^{m}(2)$, where $s$ and $m$ stand for a single-pick situation and a multiple-pick situation, respectively, and $t p$ : $t w$ represents the pick: walk time ratio. The value in the parentheses indicates the number of pickers in the system. In the single-pick situation, Gue et al. (2006) presented closed-form analytical expressions for $b_{1: 1}^{s}(2)$ and $b_{1: 0}^{s}(2)$ as shown in Table, row 1. Parikh and Meller (2010) and Hong et al. (2013) addressed multiple-pick models $\left(b^{m}{ }_{1: 1}(2)\right.$ and $\left.b^{m}{ }_{1: 0}(2)\right)$ as shown in Table, row 2.

\subsection{Comparison of Single-Pick versus Multi-Pick Models}

The following theorem shows that multiple-picks at a particular pick face produces more picker blocking in a circular-aisle abstraction for the two pick: walk ratios considered above.

Theorem 1. When the walk speed is very slow or very fast (i.e., pick: walk time $=1: 1$ and 1:0), allowing multiple-picks at a pick face always creates more picker blocking than restricting to single-pick at a pick face.

Proof. The comparison is performed for equivalent workload, i.e., equivalent number of picks per pick face. Let $u$ denote the average number of picks at a pick face. The multiple-pick model uses $u \neq p$ by definition, unlikely in the single-pick model. We use the relationship between $p$ and $u$ developed by Parikh and Meller (2009):

$$
u=\frac{p-m p^{m}+(m-1) p^{m+1}}{1-p}+m p^{m}
$$


where $m$ stands for the maximal number of picks at a pick face; $m$ is infinite in our model. Next, we simplify the relationship as $u=p /(1-p)$ or $p=u /(1+u)$ in order to compare the previous two models with Gue et al.'s models. By definition, $u$ is greater or equals 0 and less than or equals 1 , and $n$ is always greater than 1 because the single-pick situation requires spaces for two pickers. When pick: walk time $=1: 1$, Eq. (4) minus Eq. (2) is

$$
\begin{aligned}
\frac{u}{2 u+n-1+u n-u}-\frac{u(1-u)}{(n-1)\left(u^{2}+2 u+1\right)-2 u^{2}} \\
=\frac{2 u^{2}(u(n-1)+n-2)}{(u+n-1+u n)\left(u^{2} n+2 u n+n-2 u^{2}-2 u-1\right)}
\end{aligned}
$$

Because $0 \leq u \leq 1$ and $n \geq 2$, Eq. (4) minus Eq. (2) is always greater than or equals 0 . Thus, Eq. (4) is always larger than or equals Eq. (2).

When pick: walk time $=1$ : 0, Eq. (5) minus Eq. (3) is

$$
\begin{aligned}
\frac{1+u}{2+2 u+u n-u} & -\frac{1-u}{2-2 u+n u-u} \\
& =\frac{2 u^{2}(n-1)}{(u n+u+2)(u n-3 u+2)}
\end{aligned}
$$

Because $0 \leq u \leq 1$ and $n \geq 2$, Eq. (5) minus Eq. (3) is always greater than or equals 0 . Thus, Eq. (5) is always larger than or equals Eq. (3).

End of proof. Theorem 1 shows that when the work load is identical, the multiple-pick models experience heavy picker blocking. Further, note that the variation in pick density of the multiple-pick situation equals $p /(1-p)^{2}=$ $u(1+u)$, whereas the variation in pick density of the single-pick situation is $u(1-u)$. A variation of pick time at a stop should be a managerial concern for less picker blocking. However, as discussed in Section 3, a practical batch picking can occur in a narrow-aisle picking system with different batching algorithms, batch-sizing strategies, and storage policies.

\section{SIMULATION COMPARISON IN A NARROW-AISLE PICKING SYSTEM}

Our simulation study generalizes the analytical results into multiple-aisle cases, where a routing algorithm modulates the pick density to shorten the total travel distance, and repeats analysis of picker blocking over the variation of pick density. Instead of the variation of pick density, we measure the variation of the number of picks in an aisle, which explains the number of picks at a pick face as well as the routing effect.

\subsection{Experiment Design}

Using a multiple, narrow-aisle system (Figure 2) composed of a two-aisle and a ten-aisle, we compare four batching algorithms: FCFS (batches created from a sequence of orders in a first-come first-served manner); Seed (the seed algorithm developed in De Koster et al., (1999)); CW II (the Clarke and Wright algorithm (II) in De Koster et al. (1999)), see Appendix A.1 for more detail; and RBP (the heuristic route-selection-based batching procedure developed by Hong et al. (2012)), see Appendix A.2 for more detail.

The batch size is determined by the number of orders (OCS, order-capacitated batch-sizing) and the number of items (ICS, item-capacitated batch-sizing). We assume that items are stored in random locations in the two-aisle order picking system. We allow the random storage policy (RSP) and the class-based storage policy (CSP) in the ten-aisle order picking system. The classbased storage policy determines item locations using a within-aisle class-based storage policy where the A: B: $\mathrm{C}$ ratio of demand is 0.7: 0.2: 0.1. Further, class $\mathrm{A}, \mathrm{B}$, and $\mathrm{C}$ items are stored in aisles 1-2, 3-4, and 5-10, respectively. The details of the scenarios are summarized in Table 2 and the results are summarized in Section 5.3.

Since order picking profiles are diverse over industries, we consider five additional scenarios: large order size; large batch size; more pickers; faster pickers; and different size of picking system. The large order size scenarios set average order size $=$ four items (uniform [2, 6]). The item-capacitated batch-sizing scenarios set the capacity of the cart to 40 items to reflect the increase of the order size. The large batch size scenarios have 15 orders per batch in the order-capacitated batch-sizing strategy and 30 items per batch in the item-capacitated batch-sizing strategy. The increased pickers scenarios assign ten pickers and the faster pickers scenarios use pick: walk time ratio $=1: 0.1$. We also test a six-aisle order picking system. The results of the additional scenarios are discussed in Appendix A.3.

We run fifty simulations for each scenario (see the Ruben and Jacobs (1999)'s simulation model) and compare the percentage of time blocked, the number of picks in an aisle, and the standard deviation of the number of picks in an aisle (STD). Experiment profiles and parameters are summarized in Table 3.

\subsection{Correlation Analysis}

A correlation analysis to detect significance between the variation in the number of picks and picker blocking shows that the correlation coefficient of the variation in the number of picks and picker blocking is 0.73 (p-value $<2.2 \mathrm{e}-16$ and degree of freedom $(\mathrm{df})=$ 3198 ), which indicates that the variation in the number of picks strongly affects picker blocking in batch picking (see Section 4). Figure 4 plots picker blocking over 
Table 2. Two primitive order picking scenarios

\begin{tabular}{lll}
\hline & \multicolumn{1}{c}{ Two-aisle scenario } & Ten-aisle scenario \\
\hline Number of aisles & two & ten \\
Number of pick faces per aisle & 50 pick faces & 10 pick faces \\
Number of orders in a time window & 500 orders & 1,000 orders \\
Number of time windows & eight & four \\
Pick: walk time ratio & $1: 0.2$ & $1: 0.2$ \\
Number of pickers & five & five \\
Setup time & no & no \\
Capacity & ten orders or 20 items & ten orders or 20 items \\
\hline
\end{tabular}

Table 3. Experiment profiles and parameters including both primitive and additional order picking scenarios

\begin{tabular}{ll}
\hline \multicolumn{1}{c}{ Profiles } & \multicolumn{1}{c}{ Parameters } \\
\hline Algorithms & FCFS, Seed, CW II, RBP \\
Batch-sizing strategy & Order-based batch-sizing (OCS), Item-based batch-sizing (ICS) \\
Storage policy & Random storage policy (RSP), Class-based storage policy (CSP) \\
Number of aisles & two, six, ten \\
Order size & Uniform $(1,3)$, Uniform $(2,6)$ \\
Pick: walk time ratio & $1: 0.2,1: 0.1,1: 0.05$ \\
Batch size & 10 orders or 15 orders when SWP, 20 items or 30 items when PTS \\
Number of pickers & five, seven \\
\hline
\end{tabular}

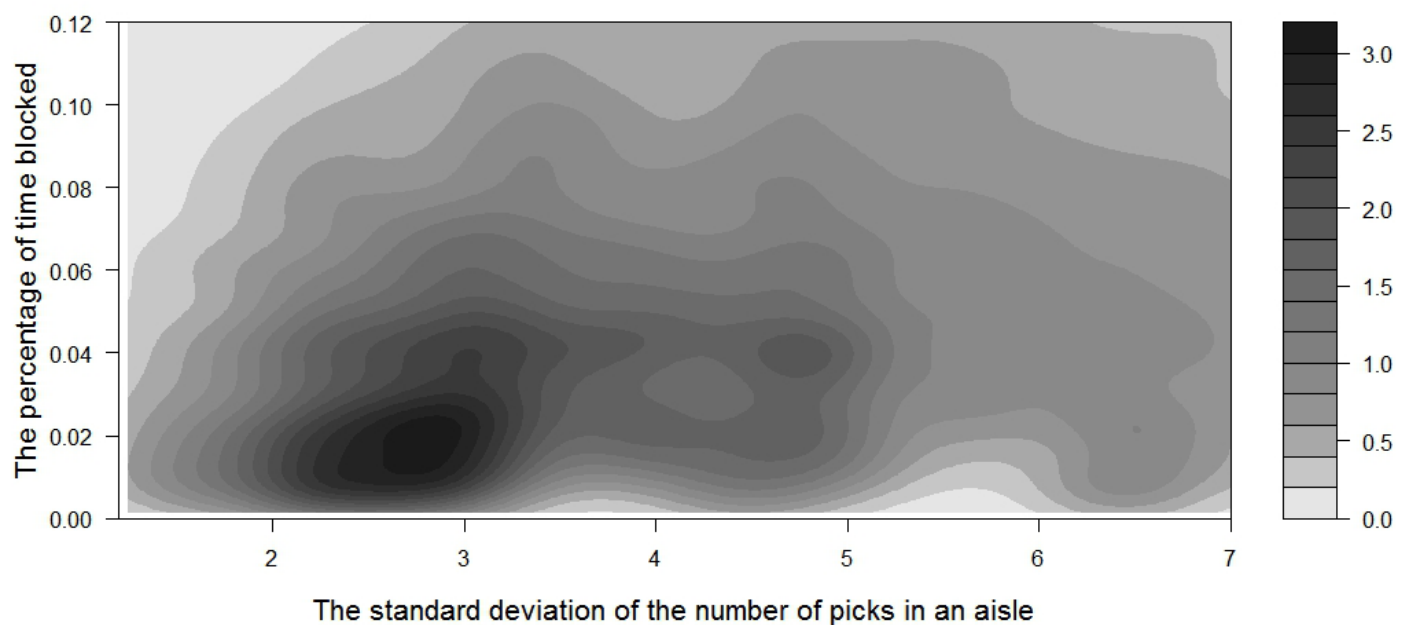

Figure 4. Correlation between "the standard deviation of the number of picks in an aisle" and "the percentage of time blocked."

the number of picks. Denser relationships, using the kernel density estimation in $\mathrm{R}$ with the default parameter for the number of points per unit area, are darker in color. The highest density area is formed from the RBP and CW II algorithms on the left and bottom of the figure. The FCSF algorithm appears on the middle of the figure. The Seed algorithm shows weak density on the right side of the relationship analysis figure.

All of the correlation coefficients in Table indicate a positive correlation between picker blocking and increased values of the experimental parameters. While most parameters result in very small differences in the correlation coefficient, the batching algorithm significantly affects the correlation coefficients.

\subsection{Summary of Observations}

We note that less picker blocking does not imply higher productivity. Thus, we review our previous results from the perspective of order picking throughput. We also consider performance in terms of the total retrieval time and the total travel distance as depicted in Figure 5. Observe that RBP and CW II are relatively efficient despite picker blocking, while Seed produces very poor results due to heavy congestion. Figure 5 illustrates the importance of selecting a batching algorithm that reduces travel distance and does not create excessive picker blocking. 
Table 4. Correlation coefficient comparison over parameters across profiles

\begin{tabular}{llccc}
\hline \multicolumn{1}{c}{ Profiles } & \multicolumn{1}{c}{ Parameters } & Correlation coefficient & df & p-value \\
\hline Algorithms & FCFS & 0.14 & 798 & $8.743 \mathrm{e}-05$ \\
& Seed & 0.05 & 798 & 0.1228 \\
& CW II & 0.65 & 798 & $<2.2 \mathrm{e}-16$ \\
& RBP & 0.60 & 798 & $<2.2 \mathrm{e}-16$ \\
\hline Batch-sizing & OBS & 0.75 & 1398 & $<2.2 \mathrm{e}-16$ \\
strategy & IBS & 0.80 & 1398 & $<2.2 \mathrm{e}-16$ \\
\hline Storage & RSP & 0.90 & 398 & $<2.2 \mathrm{e}-16$ \\
policy & CSP & 0.96 & 398 & $<2.2 \mathrm{e}-16$ \\
\hline Number & 2 & 0.96 & 398 & $<2.2 \mathrm{e}-16$ \\
of aisles & 6 & 0.95 & 398 & $<2.2 \mathrm{e}-16$ \\
& 10 & 0.90 & 398 & $<2.2 \mathrm{e}-16$ \\
\hline Order & Uniform (1, 3) & 0.90 & 398 & $<2.2 \mathrm{e}-16$ \\
size & Uniform (2,6) & 0.92 & 398 & $<2.2 \mathrm{e}-16$ \\
\hline Pick: walk & $1: 0.2$ & 0.90 & 398 & $<2.2 \mathrm{e}-16$ \\
time ratio & $1: 0.1$ & 398 & $<2.2 \mathrm{e}-16$ \\
\hline Batch size & 10 orders (OC) & 0.85 & 198 & $<2.2 \mathrm{e}-16$ \\
& 15 orders (OC) & 0.86 & 198 & $<2.2 \mathrm{e}-16$ \\
& 20 items (IC) & 0.81 & 198 & $<2.2 \mathrm{e}-16$ \\
\hline Number & 30 items (IC) & 0.94 & 198 & $<2.2 \mathrm{e}-16$ \\
of pickers & 5 & 0.89 & 398 & $<2.2 \mathrm{e}-16$ \\
\hline
\end{tabular}

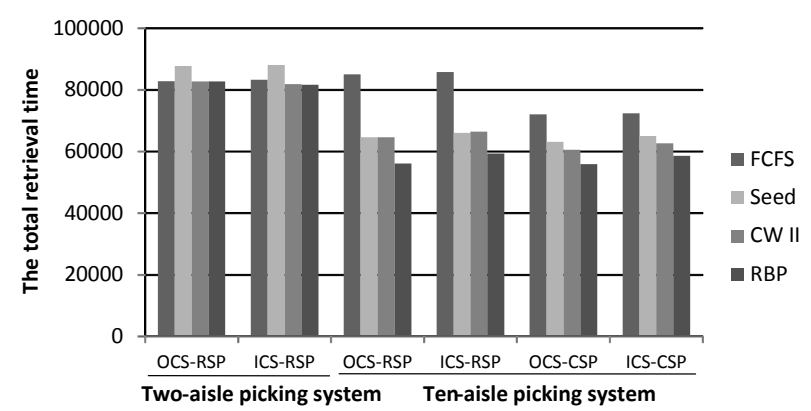

(a)

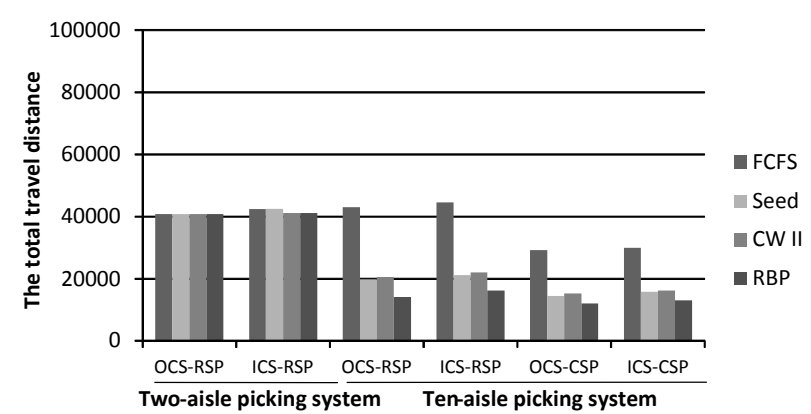

(b)

Figure 5. Comparison over four batching algorithms of: (a) total retrieval time; and (b) total travel distance.

We summarize our three findings below.

Observation 1) The batching algorithm chosen will impact picker blocking.

Intuitively, any distance-based batching algorithm could encounter significant congestion. Figure 6 details the throughput loss by the time blocked and the variation in the number of picks per aisle. As noted, the Seed algorithm creates heavy congestion compared to FCFS. However, the solutions using the CW II and RBP algorithms exhibit less congestion compared to the Seed algorithm even though the CW II and RBP's solutions are near optimal in terms of travel distance. Interestingly, the standard deviation of RBP is less than the standard deviation of the Seed algorithm, but is similar to the standard deviation of the CW II algorithm. However, RBP reduces congestion due to large reductions in the distance travelled and the relatively reasonable variation in picks per aisle.

Observation 2) The batch-sizing strategy impacts picker blocking when combined with RBP and CWII.

In the two-aisle picking system, only a single route is available under the traversal routing method. Under the item-capacitated batch-sizing strategy (ICS), as the orders are consolidated more optimally, the number of batches converges to a limiting lower bound of the number of batches and the batching algorithm reduces the 


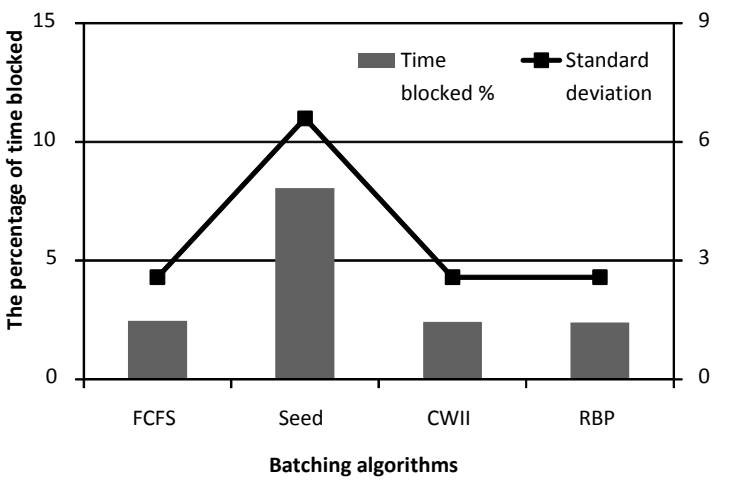

(a)

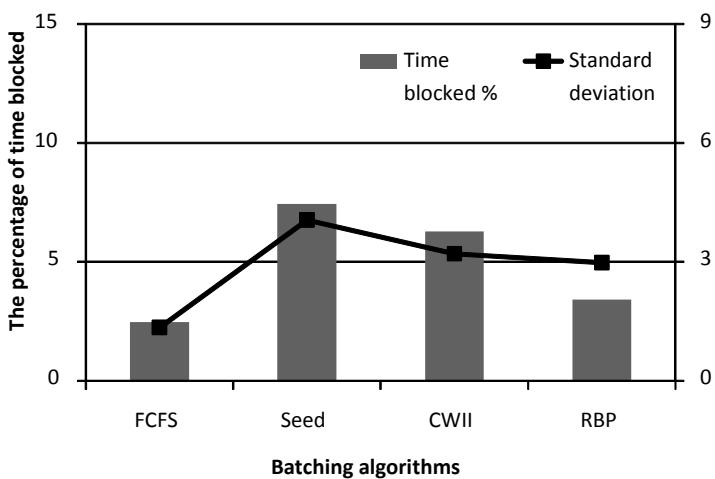

(c)

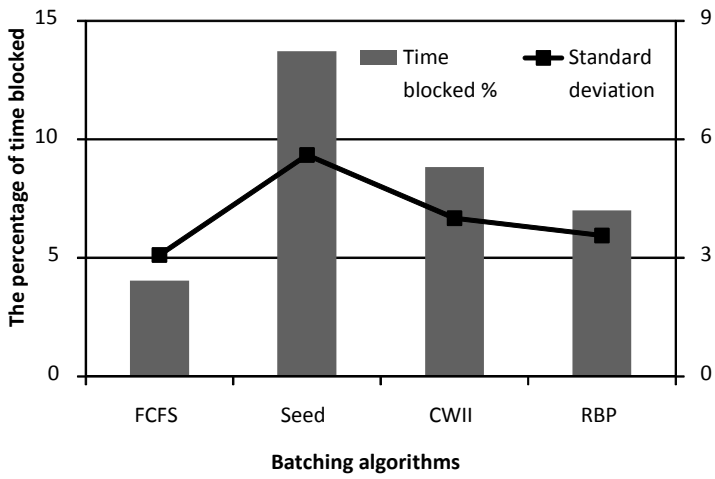

(e)

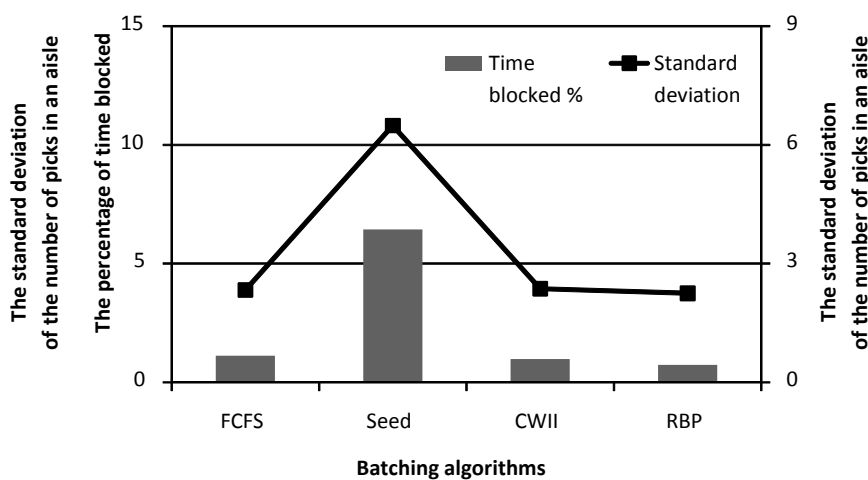

(b)
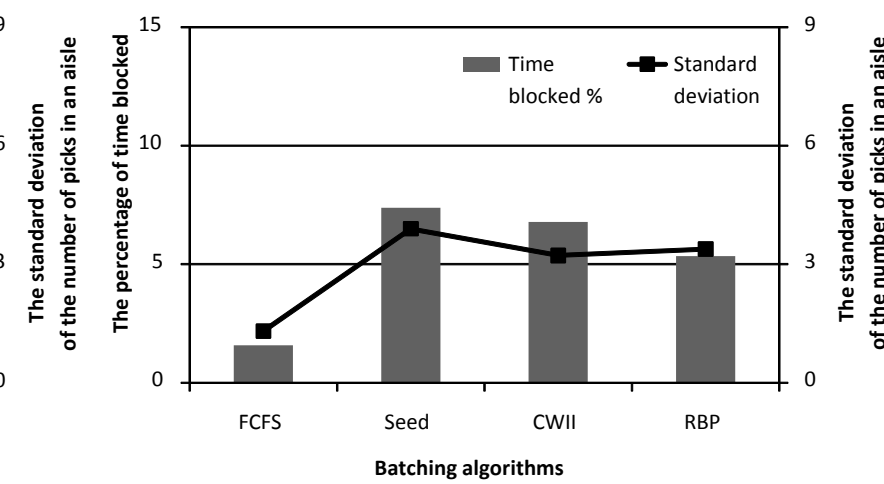

(d)
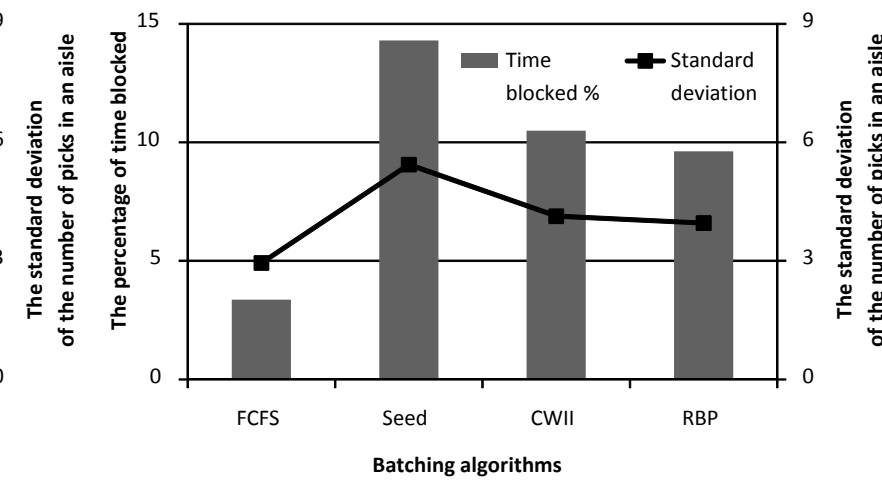

(f)

Figure 6. The percentage of time blocked and standard deviation of the number of picks per aisle over FCFS, Seed, CW II, and RBP: (a) two-aisle picking system, random storage policy, order-capacitated batch-sizing strategy; (b) two-aisle picking system, random storage policy, item-capacitated batch-sizing strategy; (c) ten-aisle picking system, random storage policy, order-capacitated batch-sizing strategy; (d) ten-aisle picking system, random storage policy, item-capacitated batch-sizing strategy; (e) ten-aisle picking system, class-based storage policy, order-capacitated batch-sizing strategy; (f) ten-aisle picking system, class-based storage policy, item-capacitated batch-sizing strategy.

variation in the number of picks across batches due to the ICS restriction. Therefore, picker blocking decreases.

In the ten-aisle picking system, the challenge is to batch orders to achieve similarity in the number of aisles visited across the batches. When the number of items in a batch (i.e., ICS) determines the batch size, the particular routing option impacts the variation in the number picks in unit distance (i.e., number of picks per aisle). The variation in the number of picks per aisle increases depending on the number of routing options. Under the OCS strategy, there is less variation in picks per aisle and a short travel distance. The OCS strategy constrains each batch to have the same number of orders, not the same number of items. The expected number of picks per 
batch will typically be proportional to the length of route, i.e., the number of aisles visited. Compared to the ICS strategy, this characteristic produce less variation in the number of picks per aisle, which reduces picker blocking.

Observation 3) Similar to Ruben and Jacobs (1999), classbased storage policy policies increase picker blocking.

Even though the RBP algorithm implements a sortwhile-pick strategy (Figure 6 (c) and (e)), the productivity loss due to congestion is $5.3-9.6 \%$. In other words, the class-based storage policy offsets the gain of the travel distance with the losses related to picker blocking (Figure 5). Observation 2 is still valid because each aisle stores items evenly under the class-based storage policy.

Our previous observations continue to hold when the order size increases, the batch size varies, additional pickers are assigned, pickers walk faster, and the number of aisles differs. RBP under the OCS strategy regulates picker blocking due to reduced variation in the time blocked. CW II scenarios under the OCS strategy and RBP scenarios under the ICS strategy also demonstrate less picker blocking.

\section{CONCLUSION AND FURTHER STUDY}

This paper investigated the relationship between picker blocking and order batching using a simple analytical comparison and several simulations. The method for constructing batches was shown to have a significant influence on the amount of picker blocking. A real batching situation confirmed that the distance-based batching method, Hong et al. (2012), combined with an appropriate sorting strategy was effective for increasing order picking throughput.

We noted that previous researchers were wary of batching approaches that minimize the travel distance because expected gains in terms of reduced travel distances could be offset by increased picker blocking. Our simulations ascertained that heavy picker blocking only occurs when batch picking is accompanied by high variations in pick density across the picking area. A conclusive simulation study showed that less variation in pick density could be achieved using a tightly packed distance-based batch formation.

Most performance evaluation models for the design and operation of a warehouse implicitly assume that the batch-sizing strategy decision (i.e., the sorting strategy) is independent of the batching method (Ruben and Jacobs, 1999; De Koster et al., 1999; Gibson and Sharp, 1992). We have suggested that picker blocking in a narrow-aisle configuration can be affected by the batchsizing strategy. When the number of aisles visited by an order picker is large, then the order-capacitated strategy improves order picking throughput by reducing picker blocking, whereas when the number of aisles is less (i.e., two-aisle picking system), the warehouse manager's best choice is an item-capacitated strategy.

Our findings have two implementable recommendations. The order-capacitated strategy combined with the batching algorithm of Hong et al. (2012) is recommended for a narrow-aisle picking system with multipleaisles. The item-capacitated strategy with an emphasis on minimizing the number of batches (i.e., packing each cart as tightly as possible with items) is recommended for situations with a single route. Even if demand increases (assuming the number of pickers is fixed, the batch size also increases) a reduction in picker blocking will occur when the batches are well-packed.

We suggest two future research directions. First, identification of a procedural method which can reduce the variation of pick density in small- and medium-scale situations is necessary. Second, explicitly modelling and controlling picker blocking offers the potential for improvements, particularly in high volume facilities.

\section{ACKNOWLEDGEMENTS}

A shorter and less technical version of this subject was published previously as "Analysis of Picker Blocking in Narrow-Aisle Batch Picking" in Progress in Material Handling Research: Proceedings of 2010 International Material Handling Research Colloquium.

We thank Andrew L. Johnson, Brett A. Peters, and Misook Ha for providing their valuable suggestions and constructive comments to improve the manuscript. This work was supported by Pusan National University Research Grant, 2014.

\section{REFERENCES}

Gue, K. R., Meller, R. D., and Skufca, J. D. (2006), The effects of pick density on order picking areas with narrow aisles, IIE Transactions, 38, 859-868.

Ruben, R. A. and Jacobs, F. R. (1999), Batch construction heuristics and storage assignment strategies for walk/ride and pick systems, Management Science, 45, 575-596.

Tompkins, J. A., Bozer, Y. A., and Tanchoco, J. M. A. (2003), Facilities Planning, J. Wiley, Hoboken, NJ.

Parikh, P. J. and Meller, R. D. (2009), Estimating picker blocking in wide-aisle order picking systems, IIE Transactions, 41, 232-246.

Parikh, P. J. and Meller, R. D. (2010), A note on worker blocking in narrow-aisle order picking systems when pick time is non-deterministic, IIE Transactions, 42, 
392-404.

Skufca, J. D. (2005), k Workers in a circular warehouse: a random walk on a circle, without passing, SIAM Review, 47, 301-314.

De Koster, R., Van der Poort, E. S., and Wolters, M. (1999), Efficient orderbatching methods in warehouses, International Journal of Production Research, 37, 1479-1504.

Gademann, N. and Van de Velde, S. (2005), Order batching to minimize total travel time in a parallelaisle warehouse, IIE Transactions, 37, 63-75.

Gademann, N., Van den Berg, J., and Van der Hoff, H. (2001), An order batching algorithm for wave picking in a parallel-aisle warehouse, IIE Transactions, 33, 385-398.

Hong, S., Johnson, A. L., and Peters, B. A. (2012), Large-scale order batching in parallel-aisle picking systems, IIE Transactions, 44, 88-106.

Clarke, G. and Wright, J. W. (1964), Scheduling of vehicles from a central depot to a number of delivery points, Operations Research, 12, 568-581.

Hong, S., Johnson, A. L., and Peters, B. A. (2013), A note on picker blocking models in a parallel-aisle order picking system, IIE Transactions, 45, 13451355.

Hong, S., Johnson, A. L., and Peters, B. A. (2015a), Quantifying picker blocking in a bucket brigade order picking system, International Journal of Production Economics.

Hong, S., Johnson, A. L., and Peters, B. A. (2015b), Order batching in a bucket brigade order picking system considering picker blocking, Flexible Services and Manufacturing.

Gibson, D. R. and Sharp, G. P. (1992), Order batching procedures, European journal of operational research, 58, 57-67.

\section{APPENDIX}

\section{A.1 Clarke and Wright II Algorithm (De Koster et al., 1999; Clarke and Wright, 1964)}

Step 1. Obtain the distance savings $s_{i j}$ for all possible order pairs $i, j$ when two orders are grouped, given the capacity of the pick device.

Step 2. Sort the savings in decreasing order.

Step 3. Select the pair with the highest savings. In the case of a tie, select a random pair.

Step 4. Combine both orders to form a new cluster if allowed by the pickers' capacity. If not, choose the next combination on the list and repeat Step 4.

Step 5. If all order combinations have not been included in a route, proceed with Step 1. In the calculation, all clusters are considered as orders. Otherwise, finish.

\section{A.2 A Heuristic Route-Packing Based Order Batching Procedure (RBP)}

RBP takes advantage of the traversal routing method. When traversal routing methods are used, all possible routes can be constructed from the warehouse layout. RBP is composed of three steps:

Step 1 . Identify potential route sets.

Step 2. Solve the route-bin packing problem (RPP) model heuristically; RPP is developed by assigning orders to routes directly, skipping the partitioning stage. Because RPP is still computationally difficult, the solution procedure considers two further computational improvements: a partial route set and a truncated branch-and-bound approach.

Step 3. Restore a feasible solution from the infeasible solution by the relaxed model.

See Hong et al. (2012) for further details. 